\title{
Comments on Injector Proton Beam Study in Run 2014
}

\author{
S.Y. Zhang
}

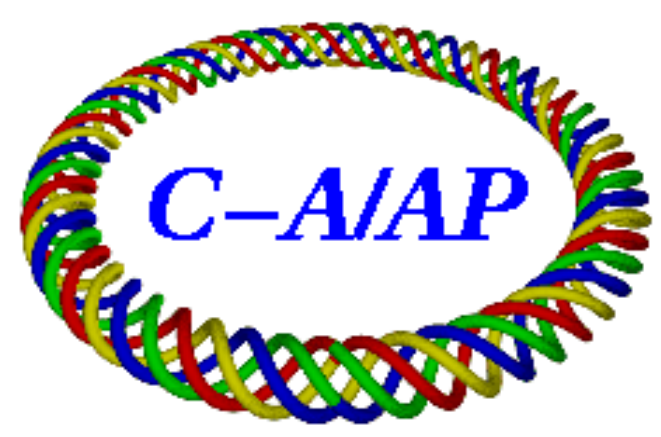

\section{Collider-Accelerator Department Brookhaven National Laboratory Upton, NY 11973}

\section{U.S. Department of Energy Office of Science, Office of Nuclear Physics}

Notice: This document has been authorized by employees of Brookhaven Science Associates, LLC under Contract No. DE-AC02-98CH10886 with the U.S. Department of Energy. The United States Government retains a nonexclusive, paid-up, irrevocable, world-wide license to publish or reproduce the published form of this document, or allow others to do so, for United States Government purposes. 


\title{
Comments on Injector Proton Beam Study in Run 2014
}

\author{
S.Y. Zhang *
}

September 15, 2014

\begin{abstract}
During the entire period of injector proton study in run 2014, it seems that the beam transverse emittance out of Booster is larger than that in run 2013. The emittance measured at the BtA transfer line and also the transmission from Booster late to AGS late are presented for this argument. In addition to this problem, it seems that the multiturn Booster injection, which defines the transverse emittance, needs more attention. Moreover, for high intensity operations, the space charge effect maybe already relevant in RHIC polarized proton runs. With the RHIC proton intensity improvement in the next several years, higher Booster input intensity is needed, therefore, the space charge effect at the Booster injection and early ramp may become a new limiting factor.
\end{abstract}

\section{Booster transverse emittance in run14 is larger than in run13}

In the entire injector proton study period of run 2014, the Booster seems to have larger transverse emittance, which had affected the study for AGS emittance and polarization.

Usually, the Booster to AGS (BtA) transfer line multiwires are used to measure the beam transverse emittance out of the Booster, where MW006 is more often used than others. From previous experiences, the horizontal beam size at MW006 is not varying too much. This is true even a heavy vertical Booster scraping applies, which may cut the vertical beam emittance at MW006 to a third, but not the horizontal. Therefore, a simple way to evaluate the Booster transverse emittance is to look at the MW006 horizontal first.

In the entire study of run 2014, the MW006 horizontal beam size has stayed larger than run 2013. This is shown in Table 1, where the MW006 horizontal beam size measurements at AGS CBM of $2 \times 10^{11}$ protons are presented for 2013 and 2014. The measured horizontal emittance at MW006 is typically at $9 \pi \mu m$ in run 2013, which is similar to previous runs starting fro run 2009, but it is more like $16 \pi \mu m$ in run 2014.

\footnotetext{
${ }^{*}$ Acknowledgement: I would like to thank H. Huang and K. Zeno for many helpful discussions
} 


\begin{tabular}{c|ccccc|ccccc}
\hline \hline & \multicolumn{4}{|c|}{2013} & \multicolumn{5}{c}{2014} \\
Time & $2 / 27$ & $2 / 28$ & $3 / 11$ & $3 / 18$ & $3 / 19$ & $5 / 14$ & $5 / 16$ & $5 / 25$ & $5 / 29$ & $6 / 2$ \\
\hline Booster input, 10 & 3.70 & 3.82 & 3.41 & 4.82 & 5.59 & 5.94 & 5.39 & 4.66 & 6.59 & 6.11 \\
AGS CBM, 10 11 & 2.16 & 2.11 & 2.07 & 1.93 & 2.10 & 2.00 & 2.09 & 2.28 & 2.18 & 2.33 \\
MW006, rms, $\sigma_{H}, m m$ & 1.40 & 1.40 & 1.48 & 1.38 & 1.44 & 1.86 & 1.88 & 1.92 & 1.81 & 1.88 \\
MW006, emittance, $\epsilon_{H}, \pi \mu m$ & 9.02 & 9.02 & 10.1 & 8.77 & 9.55 & 15.9 & 16.3 & 17.0 & 15.0 & 16.2 \\
\hline \hline
\end{tabular}

Table 1: MW006 horizontal beam emittance in run 2013 is typically at $9 \pi \mu m$ and it is $16 \pi \mu m$ in the entire injector study period of run 2014.

It has been noticed in the previous runs that, the transmission from the Booster late to AGS CBM (late) intensity is tightly related to the beam transverse emittance at the Booster and AGS.

The Booster late intensity can be reliably measured, and the AGS CBM can be easily verified with the RHIC intensity at the injection, therefore, this transmission efficiency is usually watched during the RHIC proton operations for the injector beam transverse emittance check.

In Figure 1, the entire history of AGS vertical emittance measured at the extraction in run 2013 [1] and the Booster late to AGS CBM transmission is shown. The magenta line is the average for different running period.

In the early run, with the Booster scraping ratio around 1.5, the AGS emittance is large (also the RHIC polarization is averaged $46 \%$ ), the average transmission efficiency of Booster late to AGS CBM is 78\%. After the "magic" fill 17396, the Booster scraping ratio is raised to 2.2, the AGS emittance is significantly reduced (together with the improvement of the RHIC polarization to $55 \%$ ), the Booster late to AGS CBM efficiency is raised to $85 \%$. The correlation between the beam transverse emittance and the Booster to AGS transmission is demonstrated.

The Booster late to AGS CBM efficiency in the entire proton study period of run 2014 is significantly lower than in run 2013. In Table 2, the transmission is shown at 5 different time period for the same AGS CBM intensity. Average efficiency in run 2014 is around $72 \%$, which is much lower than the later run 2013 of $85 \%$, even lower than the early run 2013 of $78 \%$.

\begin{tabular}{c|ccccc}
\hline \hline RHIC fill & 18283 & 18285 & 18296 & 18299 & 18377 \\
\hline Booster late, $10^{11}$ & 2.65 & 2.75 & 3.06 & 2.62 & 3.00 \\
AGS CBM, $10^{11}$ & 2.00 & 2.00 & 2.00 & 2.00 & 2.00 \\
Transmission, \% & 75 & 73 & 65 & 76 & 67 \\
\hline \hline
\end{tabular}

Table 2: The Booster late to AGS CBM transmission efficiency in 5 different times of run 2014 injector proton study, averaged at $72 \%$. The RHIC fill is used to indicate the study time.

In Figure 2, the study at the RHIC fill 18377, which is at the end of the proton study period, is shown. The Booster late to AGS CBM efficiency stayed at less than $70 \%$.

Small transverse emittance is a necessary condition for high transmission efficiency from Booster to AGS injection and ramp. The low Booster to AGS transmission in run 2014 is agreeable with the argument that the transverse emittance at the Booster in run 2014 is larger than run 2013. 

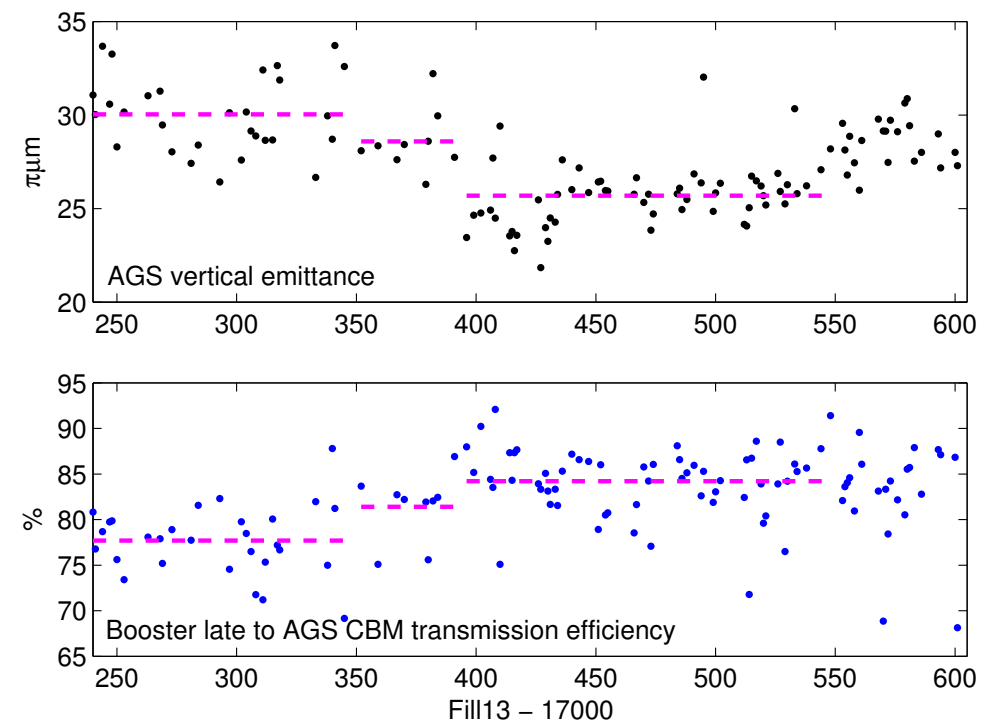

Figure 1: The entire history of AGS vertical emittance measured at the extraction and the Booster late to AGS CBM transmission is shown for run 2013. The correlation between the beam transverse emittance and the Booster to AGS transmission is demonstrated.
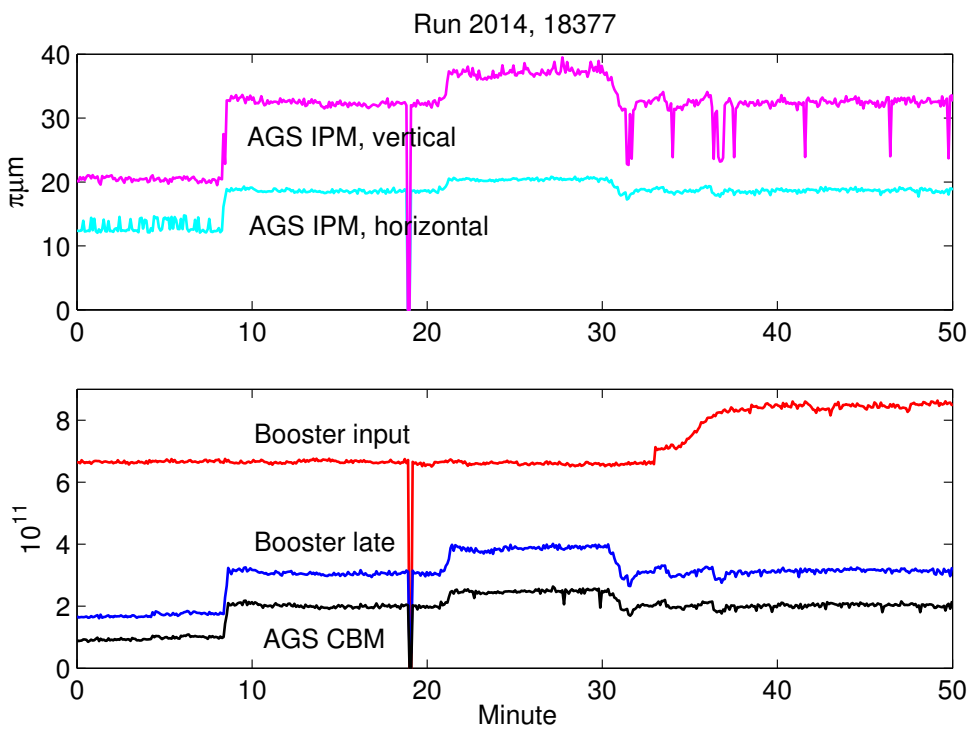

Figure 2: The study at fill 18377, which is at the end of the proton study period, is shown. For AGS CBM around $2 \times 10^{11}$, the Booster late to AGS CBM efficiency stayed at less than $70 \%$. 

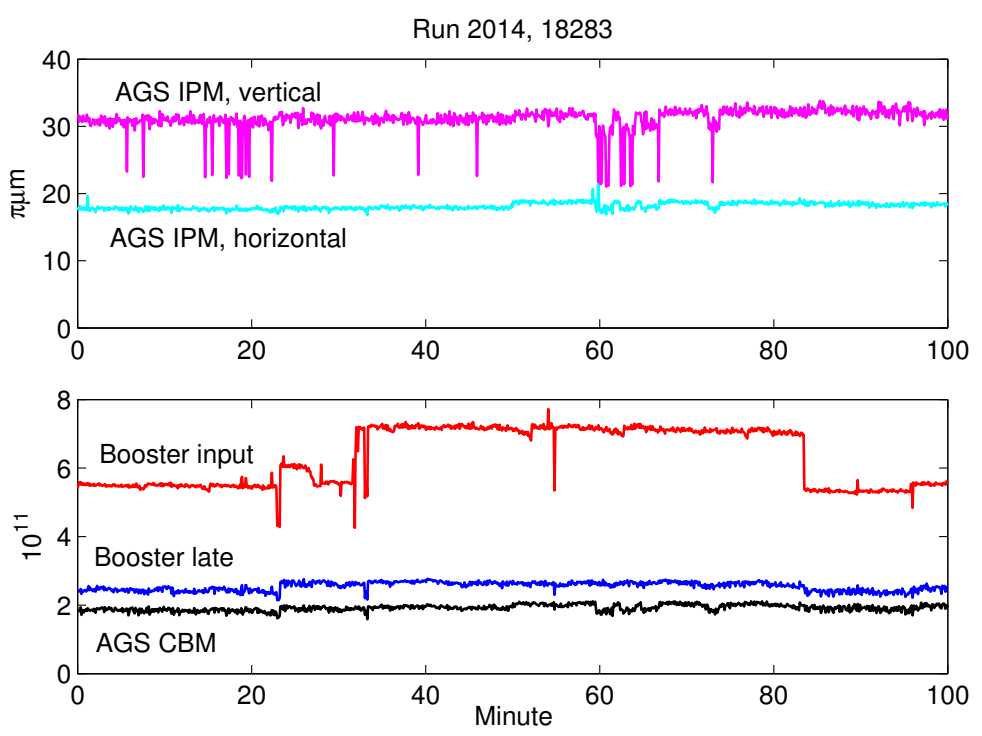

Figure 3: Study by extending the LINAC Tank 9 pulse width for $300 \mu s$ to $400 \mu s$ is shown.

The large Booster transverse emittance may come from two sources, one is a large emittance "defined" during the Booster multiturn injection, where the LINAC beam emittance, the Booster injection and RF capture, are relevant, and another is the emittance growth in the Booster.

The injector is a key for further RHIC polarized proton intensity improvement. For injector proton intensity, emittance, and polarization improvement, the Booster injection and early $\mathrm{RF}$ capture might be a most critical place.

\section{Transverse emittance defined at the Booster injection and RF capture}

A brief study by extending the LINAC Tank 9 pulse width from $300 \mu s$ to $400 \mu s$ is performed in run 2014. By extending the pulse width, more beam is injected into the Booster, and therefore, more Booster scraping can be applied for given AGS intensity.

The study is shown in Figure 3, where the Booster input, late intensities and the AGS CBM intensity are presented together with the AGS IPM emittance measurement. In the middle of the study period, the injection pulse width is extended to $400 \mu \mathrm{s}$, which gives rise to $25 \%$ higher Booster input intensity. For the same AGS CBM intensity, the Booster scraping is increased, but the AGS emittance stays unchanged.

With $100 \mu \mathrm{s}$ longer injection period at the Booster, some 84 more turns is added at the injection. In the study, it seems that these additionally injected particles are just stacked in at large emittance, and almost all are scraped off later in the Booster ramp.

The current Booster injection has been developed at $300 \mu s$ LINAC pulse width, started from early 2009. Issues relevant to the transverse emittance "defined" at the end of the multiturn injection include the LINAC beam parameters, LINAC to Booster optics matching, the Booster 

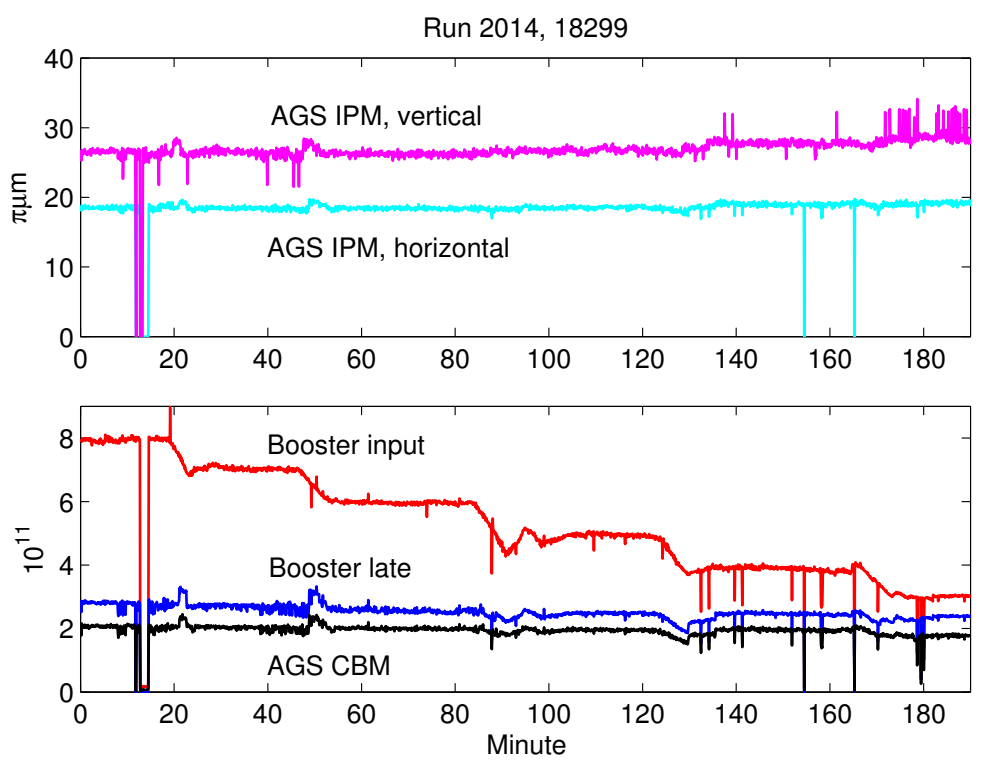

Figure 4: Proton source Rb temperature is adjusted for study.

magnetic field, the injection bump, the $H^{-}$charge exchange stripping foil passing, horizontal and vertical tunes and chromaticity, and the orbit.

It would be expected that to extend the $300 \mu s$ injection to $400 \mu s$ injection require significant attention on the tuning. In the successful run 2006, the LINAC pulse width settled and stayed at $400 \mu s$ only after several weeks of intensive machine tuning, by not only looking at the Booster and AGS, but also the improvement of RHIC beam intensity, emittance, and polarization. The RHIC improvement offers more reliable verification on Booster injection tuning.

\section{$3 \quad$ Emittance growth associated with the Booster injection and RF capture, the space charge}

By pushing the Booster input intensity beyond $5 \times 10^{11}$, with both the OPPIS tuning and the raised source $\mathrm{Rb}$ temperature, the space charge effect seems to become relevant in the transverse emittance growth in the Booster, especially at the injection and the RF capture.

In fact, the AGS high intensity proton operation prior to the RHIC era is significantly affected by the space charge effect at the Booster injection [2-4]. With the very high proton intensity, the space charge force had effectively blow-up the transverse emittance at the injection, filling the machine acceptance with the normalized emittance of $60 \pi \mu \mathrm{m}$. The machine acceptance only cleared up more than $10 \mathrm{~ms}$ after the injection at about $350 \mathrm{MeV}$ (beam injected at $200 \mathrm{MeV}$ ) with the emittance of $80 \pi \mu m$. During this period, up to $40 \%$ beam is lost.

Under this situation, even the adjustment of the Booster beam orbit at the injection is not sensitive to the beam loss. Attention is paid to the longitudinal painting, which may make longer bunch with lower peak current, and hence smaller space charge force for the entire and/or a part 
of the bunch. Each improvement of the bunch length immediately reduces beam loss at the early Booster ramp.

To evaluate the space charge force in the current Booster injection, the beam intensity, the transverse emittance, and the bunch length at the injection and RF capture are the relevant parameters

The Booster injection setting in 2014 study is similar to run 2013. After the multiturn stacking, the RF capture is performed with a rising magnetic field and the RF voltage as well. At the end of the RF capture, the bunch length is shortened, the peak current is high, and the space charge force reached a peak. After that, with the ramping, the space charge force is reduced gradually.

It is estimated [5] that at that point, the Booster magnetic field is 0.176 Tesla and the RF voltage is $24.3 \mathrm{kV}$. Nevertheless, the bunch length in the Booster ramp is not measured in run 2014 study.

In run 2013, a bunch length measurement was performed at a point close to the extraction, which is shown in Table 3. The bunch length of about $120 \mathrm{~ns}$ and the RF voltage of $23 \mathrm{kV}$ implies that the longitudinal emittance of $0.66 \mathrm{eVs}$.

Assuming the longitudinal emittance is unchanged at $0.66 \mathrm{eVs}$, and using the same RF voltage of $23 \mathrm{kV}$, the bunch length is projected as $296 \mathrm{~ns}$ at the end of the capture, which is at $89.9 \mathrm{~ms}$ in the cycle.

To estimate the space charge force, taking the Booster intensity at $1 \times 10^{12}$, the transverse emittance at $9 \pi \mu \mathrm{m}$. The peak current is $2.10 \mathrm{~A}$ and $0.86 \mathrm{~A}$, and the space charge tune spreads are $\Delta \nu_{i n c}=0.137$ and $\Delta \nu_{i n c}=0.480$, for the extraction and the end of the capture, respectively. The space charge calculation is following [6], and the relevant parameters are shown in Table 3.

\begin{tabular}{c|cccccccccc}
\hline \hline & $B$ & $\beta / \gamma / \eta$ & $V_{r f}$ & $f_{r f}$ & $N$ & $\epsilon_{N, 95 \%}$ & $\tau_{\ell}$ & $I_{\text {peak }}$ & $\epsilon_{\ell}$ & $\Delta \nu_{\text {inc }}$ \\
& $T$ & & $k V$ & $M H z$ & $10^{12}$ & $\pi \mu m$ & $n s$ & $A$ & $e V s$ & \\
\hline 2013 extraction & 0.521 & $0.917 / 2.510 /-0.116$ & 23 & 1.363 & 1 & 9 & 120 & 2.10 & 0.66 & 0.137 \\
$201489.9 \mathrm{~ms}$ & 0.176 & $0.617 / 1.270 /-0.577$ & 23 & 0.916 & 1 & 9 & 296 & 0.86 & 0.66 & 0.480 \\
\hline \hline
\end{tabular}

Table 3: The bunch length at $89.9 \mathrm{~ms}$ is projected by the measurement of extraction. The calculated space charge tune spread at the extraction is not a big problem, but it is of concern at the end of the capture.

It is noted that the space charge tune spread at the extraction, 0.137, maybe not a problem, but at the low energy right after the capture, 0.480, it is rather large.

It is also noted that the intensity of $1 \times 10^{12}$ is higher than that achieved in run 2014 , but the transverse emittance of $9 \pi \mu m$ is larger than the LINAC emittance estimated of $4.75 \pi \mu m$ in run 2014, and much larger than the $3.5 \pi \mu m$ in run 2013 [7].

How to evaluate the effect of the space charge is another issue, for that the high intensity run 1998 can be used as a reference [4]. The detailed comparison of 1998 high intensity Booster injection and the 2014 Booster injection with RF capture are shown in Table 4. 


\begin{tabular}{c|cccccccccc}
\hline \hline & $B$ & $\beta / \gamma / \eta$ & $V_{r f}$ & $f_{r f}$ & $N$ & $\epsilon_{N, 95 \%}$ & $\tau_{\ell}$ & $I_{\text {peak }}$ & $\epsilon_{\ell}$ & $\Delta \nu_{\text {inc }}$ \\
& $T$ & & $k V$ & $M H z$ & $10^{12}$ & $\pi \mu m$ & $n s$ & $A$ & $e V s$ & \\
\hline $201489.9 \mathrm{~ms}$ & 0.176 & $0.617 / 1.270 /-0.577$ & 23 & 0.916 & 1 & 9 & 296 & 0.86 & 0.66 & 0.480 \\
1998 injection & 0.155 & $0.566 / 1.213 /-0.636$ & 45 & 0.841 & 15 & 60 & 900 & 5.36 & 4.90 & 0.461 \\
\hline \hline
\end{tabular}

Table 4: Space charge force comparison of high intensity AGS operation in 1998 and in RHIC polarized proton runs.

The Booster injection in 1998 is with very high intensity of $15 \times 10^{12}$, but the transverse emittance is filling up the machine acceptance of $60 \pi \mu \mathrm{m}$. The Booster injection was with the longitudinal painting, using the fast chopper, second rf harmonic, rising $\dot{B}$ and rf voltage, that gave rise to the bunch length $75 \%$ of the machine circumference, at $900 \mathrm{~ns}$ [4, Figure 3].

For 1998 high intensity AGS run and the current RHIC polarized proton run, the longitudinal and transverse emittance, the intensity and peak current, are very different at the Booster injection. However, if the Booster input intensity in the current run is pushed to $1 \times 10^{12}$, even with the transverse emittance of $9 \pi \mu m$ at the injection, the space charge tune spreads become comparable.

For the typical Booster input intensity used starting from run 2009 to run $2013,0.4 \times 10^{12}$ to $0.5 \times 10^{12}$, taking the transverse emittance of $9 \pi \mu m$, the space charge tune spread is 0.20 to 0.24 . It is still modest compared with the high intensity AGS run in 1998, but maybe not completely out of the concern for possible emittance growth at the Booster injection.

In the next several years, the RHIC intensity would be pushed to $3 \times 10^{11}$, the Booster input intensity has to be pushed higher, say, $1 \times 10^{12}$, and the space charge is an issue to deal with.

Following are some questions of interest.

1. The transverse emittance of the LINAC beam at the Booster injection, which is very important for the Booster emittance improvement. Also the momentum spread of the LINAC beam, which is important for the RF capture.

2. The transverse emittance defined at the multiturn Booster injection. Intensive tuning may be needed for reducing the emittance growth at the injection.

3. The RF capture after the injection. Intensive tuning may be needed for making a longer bunch, hence to reduce the space charge effect. Adding the second rf harmonic might be the first step.

4. Booster early ramp. Currently, the ramp is started together with the RF capture, further optimization may be needed.

5. The evolution of the horizontal and vertical emittance in the Booster ramp. It is not clear how the horizontal and vertical emittance evolute at the Booster injection and ramp.

6. The effect of the Booster scraping. Vertical and horizontal scraping at different stages of the Booster ramping may be tested for optimization. 


\section{References}

[1] V. Schoefer, "Managing Emittances: Transverse and Longitudinal", RHIC retreat 2013.

[2] S.Y. Zhang, "Simulation of Booster Proton Injection - Longitudinal" , AGS/AD/Tech. note, No. 480, Aug. 1998.

[3] S.Y. Zhang, "High Intensity Proton Injection at the Booster", C-A/AP/8, Jan. 2000.

[4] S.Y. Zhang, L.A. Ahrens, J.M. Brennan, T. Roser, "AGS Booster High Intensity Proton Beam Injection", 9th ICFA Mini-Workshop on High Intensity High Brightness Hadron Beams, CERN, Geneva, Switzerland, March 2000.

[5] K. Zeno, private communications.

[6] S.Y. Zhang, T. Roser, W.T. Weng, "Calculation of Incoherent Space Charge Tune Spread", AGS/AD/Tech. note, No. 449, Oct. 1996.

[7] H. Huang, "AGS/Booster pp Study", RHIC Retreat 2014. 